\title{
Nanoscopic Domains in Model and Bacterial Membranes
}

\author{
John Katsaras \\ Large Scale Structures Group, Neutron Sciences Directorate and Shull Wollan Center, Oak Ridge \\ National Laboratory, Oak Ridge, TN, 37831, United States
}

Biomembranes are sophisticated and dynamic machines that perform a diverse array of functions, including selective transport, localization, communication and recognition, to name a few. It is also widely accepted that the plasma membrane is laterally heterogeneous containing nanoscopic regions enriched in certain types of lipids, which have different physical properties from the surrounding lipids. These functional lipid domains of lipids and proteins are commonly referred to as "rafts", and have been implicated in a wide range of cellular functions, including signal transduction, drug uptake, and interactions with pathogens. In recent years, we have used small angle neutron scattering and neutron spin echo to study nanoscopic lipid domains in model membrane systems, and more recently in the Gram-positive bacterium Bacillus subtilis, in which the first ever observation of lipid domains was made. Data from these studies will be presented. 